\title{
NON-LIVER TRANSPLANTATION
}

31

\section{LAPAROSCOPIC CYSTOPERICYSTECTOMY}

N Gupta, A Javed, AK Agarwal

Department of Gastrointestinal Surgery, GB Pant Hospital and MAM College, New Delhi, India

Background: Hydatid disease of the liver is endemic in India and is a common health problem. Radical surgery (cystopericystectomy or formal resection) is the treatment of choice as it reduces the incidence of recurrence and also decreases the incidence of cavity-related complications. This is often performed by conventional open surgery.

Aim: To analyze our experience of radical resectional surgery for hydatid disease of the liver performed laparoscopically. Method: We analyzed the prospectively collected data on hydatid cyst of the liver who were managed at our center with laparoscopic radical surgery (cystopericystectomy, formal liver resection).

Result: Eight patients underwent laparoscopic resection for hydatid cyst of liver. There were 2 males and the mean age of the patients was 34.5 years (23-56 years). Pain was the presenting complaints in these patients. One patient had pre-operative jaundice and underwent pre-operative stenting. In 3 patients, the cyst was located in the right lobe, in 1 it was involving both lobes and in the rest 4 patients, it was present in the left lobe. Three patients had Gharbi type III cysts, 2 patients had type II, and 3 patients type I cyst. PAIR was planned for 2 patients; however, it was abandoned in both, one due to cystobiliary communication and aspiration of thick pultaceous material in the other patient. Average sizes of the cysts were $7 \mathrm{~cm}$. Three patients underwent laparoscopic left lateral hepatectomy and 5 patients underwent total closed cystopericystectomy. In none of the patients, any spillage of contents occurred. Mean duration of surgery was $240 \mathrm{~min}(180-300 \mathrm{~min}$ ) and the average blood loss was $112.5 \mathrm{~mL}(50-250 \mathrm{~mL})$. No patient had postoperative bile leak and the average postoperative hospital stay was 7.6 days (3-12 days).

Conclusion: Radical resection is a well-established surgical procedure for hydatid cyst of liver. It can be safely performed laparoscopically in selected group of patients with excellent results.
32

\section{PORTAL VEIN THROMBUS IRRADIATION- AN ALTERNATIVE IN INOPERABLE HEPATOCELLULAR CARCINOMA}

A Abhishek, T Kataria, K Sharma, KP Karrthick, K Madan, T Piplani

Cancer Institute, Medanta-The Medicity, Gurgaon, India; Institute of Liver and Biliary Sciences (ILBS), New Delhi, India

Background: Portal vein tumor thrombosis (PVTT), in a case of hepatocellular carcinoma (HCC), is considered poor risk and has been reported to be associated with unfavorable outcomes to the established treatment regimens like surgical resection or TACE (transarterial chemoembolization). Radiotherapy (RT) has shown survival benefits and promises to be a valid salvage therapy in such cases. Aim: To review and establish the role of RT in advanced HCC with portal venous thrombosis.

Materials and Method: Literature was reviewed for the role of radiotherapy in PVTT along with the case selection criteria, technique, expected benefits, and possible side effects of the treatment.

Discussion: Definitive treatment strategy is not established for PVTT in advanced HCC. With $34-84 \%$ incidence, PVT cannot be overlooked and demands alternative approaches. Results of surgery in such cases are dismal and palliative chemotherapy (TACE) may increase the risk of ischemic events. In such cases, radiotherapy has been widely reported to have an objective response rate of $37.5-57.9 \%$, with a median survival time of 6.7-10.7 months. Post PVTTRT, re-canalization may be achieved in $60-75 \%$ cases and re-considered for TACE/primary management with acceptable outcomes. Therefore, RT is a promising salvage alternative in PVTT cases not fit for conventional treatment modalities.

Medanta Experience: We recently treated a 54-year-old gentleman, diagnosed to have PVTT with HCC, with 60 Gy in $20 \mathrm{Fr}$ by a volumetric modulated arc therapy (VMAT) using respiratory motion management. He tolerated the therapy well and evaluation of CT scans 2 months postRT showed necrosis at tumor thrombus site. He has been re-started on oral chemotherapy and is on close follow-up.

33

\section{HEPATIC RESECTION IN CHILDREN-} TWENTY-ONE CASES

\author{
S Shrotriya \\ Department of Surgery, KEM Hospital, Pune, India
}

Aim: To assess the indications, technique, complications, and outcome of pediatric hepatic resections. 
Method: Twenty-one patients were included in this series from the age group ranging from 6 days to 5 years. All were diagnosed to have a liver tumor clinically, on ultrasonography (USG) and computed tomography (CT) scan. Biopsy was done in 10 patients and in all $\alpha$-fetoprotein (AFP) levels were done. All were subjected to surgical resection either directly or after 2-3 cycles of chemotherapy. Postoperative care was given to all. On follow-up, AFP levels were repeated and USG was done. Maximum follow-up was 5 years.

Result: Eighteen patients survived. Three died in perioperative period, one required re-exploration, one had bile leak, and one had wound infection. There were no recurrences in our series.

Conclusion: Pediatric liver resections are needed mostly for hepatoblastoma and hemangioendothelioma. AFP levels are always raised and are good prognostic markers as well. Pre-operative chemotherapy is indicated in large or inoperable tumors. Major complications like hemorrhagic shock, air embolism, and bile leak can be fatal. Improving the results is possible with specialized training, improved pre-operative and intra-operative imaging and better ICU care. There is a significant learning curve in doing successful liver resections in children.

\section{4}

\section{CYSTOPERICYSTECTOMY-PATIENT SELECTION IS ESSENTIAL}

A Mehta, H Parekh, D Vasavada

Department of Radiodiagnosis and Department of Surgery, MP Shab Medical College, Jamnagar, India

Background: Cystopericystectomy is one of the major hepatic resections for benign lesion, needs proper selection of patient in comparison with partial cystectomies for hydatid disease.

Aim: To compare the feasibility of cystopericystectomy with conventional partial cystectomies for hydatid cysts of liver.

Materials and Method: We present a study of liver hydatid cysts over a span of 6 years, where patients were admitted in a secondary healthcare setup of district level hospital. Patients' age ranged from 6 years to 65 years, with some of them having comorbid conditions such as diabetes mellitus, chronic obstructive lung disease, and ischemic heart disease. We included 112 patients out of whom 102 were subjected to conventional partial cystectomies and 10 had undergone liver resection in the form of cystopericystectomy. We compared the outcomes in various categories including the time required for surgery, ease of operation, blood requirements, complications, recurrence, morbidity, and mortality.

Result: Both of these procedures were equally effective as far as cyst clearance was concerned, as we have not seen recurrence in this period of 6 years. However, partial cystectomies scored over cystopericystectomy as far as timing, ease of operation, and blood requirements were concerned. We had one biliary fistula in partial cystectomy and one death due to torrential bleed from middle hepatic vein in the cystopericystectomy group.

Conclusion: Cystopericystectomy should be considered in patient fit for major liver resection without any comorbidity and it can be kept reserved for infected hydatid cysts, where biliary communication is difficult to handle in partial cystectomy.

\section{5}

\section{ETIOLOGY OF RENAL DYSFUNCTION IN CIRRHOSIS}

\author{
R Baijal, K Praveen, M Jain, D Gupta, N Shah \\ Department of Gastroenterology, Jagjivanram Hospital, \\ Mumbai, India
}

Background: Renal dysfunction in cirrhosis may be due to prerenal or renal causes or due to hepato-renal syndrome. It is a poor prognostic marker and has a high mortality.

Aim: To determine the etiology of renal dysfunction in cirrhosis and its outcome.

Method: Patients with cirrhosis admitted in the last 1 year were included. All underwent routine investigations including hemogram, liver profile, blood urea nitrogen, creatinine, chest X-ray, and urine routine. Patients with renal dysfunction were defined as serum creatinine $>1.5 \mathrm{mg} / \mathrm{dL}$. They were further evaluated with abdominal ultrasonography, urine sodium/potassium, urine albumin; 24 hour urinary albumin, serum IgA, C3, and C4 levels when necessary. Patients were divided into infection-related renal failure; hypovolemia-induced renal failure, intrinsic renal dysfunction, and hepato-renal syndrome (HRS) (not due to infection).

Result: One-hundred and thirty-five (119 males) patients were included. The etiology of cirrhosis was alcohol 95, hepatitis B 13, non-alcoholic steatohepatitis (NASH) 10, cryptogenic 7 , hepatitis $C 6$, autoimmune hepatitis $(\mathrm{AIH})$ 3 , and hepatitis $\mathrm{B}+$ hepatitis $\mathrm{C} 1$. Mean model for end stage liver disease (MELD) score was $15 \pm 3$. Renal dysfunction was noted in 46 patients (35\%). Infection-related renal dysfunction occurred in 20 (43.48\%), hypovolemia 16 (34.78\%), renal disease 7 (15.22\%), and HRS (not due to infection) in 3 (6.5\%), (2 expired and 1 underwent transjugular intrahepatic porto-systemic shunt [TIPSS]). Renal dysfunction because of infection was due to spontaneous bacterial peritonitis 14 , pneumonia 3 , urinary tract infection (UTI) 2 and cellulitis 1. Renal dysfunction because of hypovolemia was due to gastrointestinal blood loss 8 , diuretics 6 and diarrhea 2. Cause of intrinsic renal disease was NASH (diabetic nephropathy) 3, alcoholic (IgA nephropathy) 2, and viral hepatitis (glomerulonephritis) 2 . Mortality in patients with renal dysfunction was 16 out of 